\title{
Superficial siderosis of the central nervous system: secondary progression despite successful surgical treatment, mimicking amyotrophic lateral sclerosis. Case report and review
}

\author{
Michael Payer • Cyrille Sottas • Christophe Bonvin
}

Received: 24 November 2009 / Accepted: 1 April 2010/Published online: 23 April 2010

(C) Springer-Verlag 2010

\begin{abstract}
Superficial siderosis of the central nervous system is a rare disorder with hemosiderin deposition in the spinal and cranial leptomeninges and subpial layer, mostly from repetitive subarachnoid hemorrhage. Progressive sensorineural deafness, cerebellar ataxia, and pyramidal signs comprise the typical clinical presentation. We describe a 47-year-old patient, who showed initial 2-year improvement after successful occlusion of an intradural bleeding source at T4. Secondary progression of symptoms without further bleedings was noted thereafter, with a clinical picture of amyotrophic lateral sclerosis. This case illustrates that the disease may progress secondarily even without re-bleedings, and that secondary progression might be due to a similar pathomechanism as in amyotrophic lateral sclerosis.
\end{abstract}

Keywords Superficial siderosis - Central nervous system · Recurrent subarachnoid hemorrhage $\cdot$ Spinal intradural hemorrhage Amyotrophic lateral sclerosis

\footnotetext{
M. Payer

Department of Neurosurgery, University Hospital of Geneva, Geneva, Switzerland

C. Sottas $\cdot$ C. Bonvin

Department of Neurology, University Hospital of Geneva, Geneva, Switzerland

M. Payer $(\bowtie)$

Department of Neurosurgery, Faculty of Medicine, University

Hospital of Geneva,

Rue Gabrielle-Perret-Gentil 4,

1211 Geneva 14, Switzerland

e-mail: mpayer@hotmail.com
}

\section{Introduction}

Superficial siderosis (SS) of the central nervous system (CNS) is a rare disorder with hemosiderin deposition in the spinal and cranial leptomeninges and subpial layer, usually resulting from chronic subarachnoid hemorrhage $[2,5,6$, $13,21,26]$. The clinical course is progressive with a typical triad of sensorineural deafness, cerebellar ataxia, and pyramidal signs, and the prognosis is catastrophic [6]. In about half of the patients, no source of hemorrhage can be identified, while in the other half of the patients, dural pathologies, cervical nerve root avulsions, vascular tumors, and vascular anomalies have been found as bleeding sources $[1,6,8]$. We describe the surgical management and clinical course in a case, in which a vascular anomaly was found on an intradural nerve root at the level of the fourth thoracic vertebra (T4).

\section{Case report}

A 47-year-old woman, in good health except for a noninsulin-dependent diabetes, presented with progressive bilateral hypoacusia, cerebellar ataxia, and tetraparesis over 7 years. No relevant trauma or acute symptom onset was reported. On admission, the patient was able to walk with crutches, and the neurological examination showed muscle cramps, fasciculations, diffuse amyotrophy, spastic tetraparesis, and cerebellar ataxia. Hypacusia, ageusia with normal olfaction, minor dysarthric speech, and dysphagia were observed.

MRI of the entire neuroaxis showed signs of hemosiderin deposition on the cerebral and cerebellar surface (Fig. 1), an intradural fluid collection in the anterior cervical and thoracic spine, a non-aggressive vertebral hemangioma in 
Fig. 1 Axial T2-weighted cranial MRI, demonstrating hypointense margins around the mesencephalon (arrow) and cerebellar hemispheres (arrow) as signs of leptomeningeal hemosiderin deposition

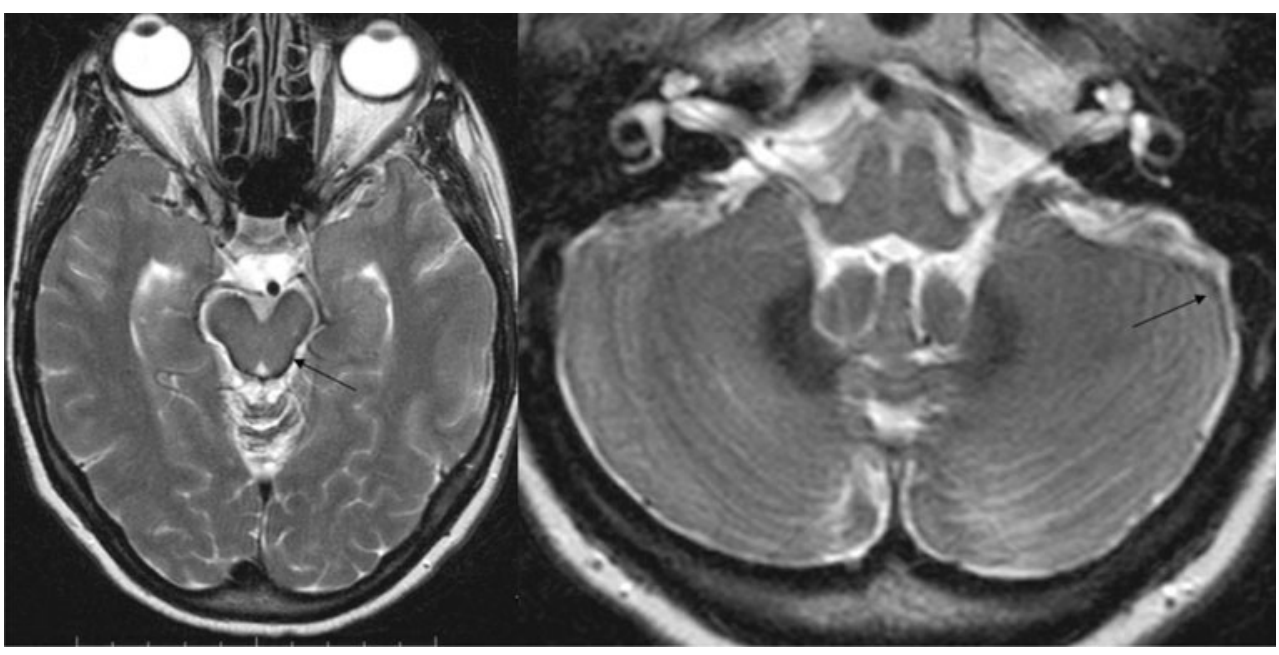

the body of T4, and an intradural calcification posterior the T4 vertebral body, without contact to the vertebral hemangioma (Fig. 2). CT scan additionally confirmed the calcification posterior to and separated from the T4 vertebral body (Fig. 3). Spinal angiography was normal. Cerebral spinal fluid (CSF) analysis was strictly normal except for elevated ferritin levels $(43.1 \mu \mathrm{g} / \mathrm{l})$.

As the bleeding source was suspected to be located within or in close contact with the intradural calcification

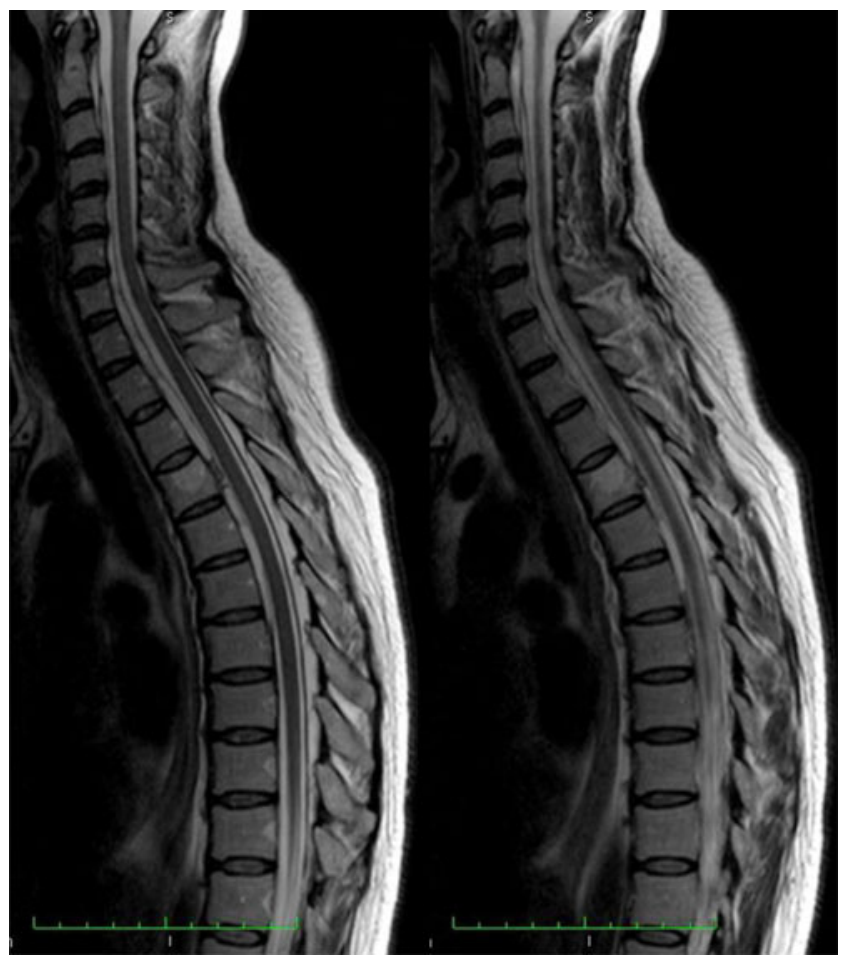

Fig. 2 Sagittal T2-weighted cervico-thoracic median and paramedian MRI images showing an intradural fluid collection in the anterior cervical and thoracic spine, a non-aggressive vertebral hemangioma in the body of T4 and an intradural calcification posterior the T4 vertebral body, without contact to the vertebral hemangioma posterior to the T4 vertebral body, surgical exploration was proposed to and accepted by the patient.

Surgery

Under general anesthesia, the patient was positioned in the prone position with the operating table slightly rotated to the lift up the right side. The skin was incised vertically in the midline with a horizontal counterincision on the right. A right-sided costo-transversectomy at $\mathrm{T} 4$ and $\mathrm{T} 5$ and a rightsided laminectomy T3-T5 was performed, which gave extrapleural access to the lateral convexity of the vertebral body of T4. The discs T3/4 and T4/5 were excised, and right-sided hemi-corpectomy of $\mathrm{T} 4$ was performed. The $\mathrm{T} 4$ nerve root was sectioned extradurally. Under the microscope, the dura was opened sagittally just ventral to the T4 root, and water-clear fluid was drained spontaneously from the subarachnoid space. A thickened arachnoid membrane became visible, and the expected anvil-like calcification was found between the thickened arachnoid and dura (Fig. 4). It was mobilized with a microdissector and

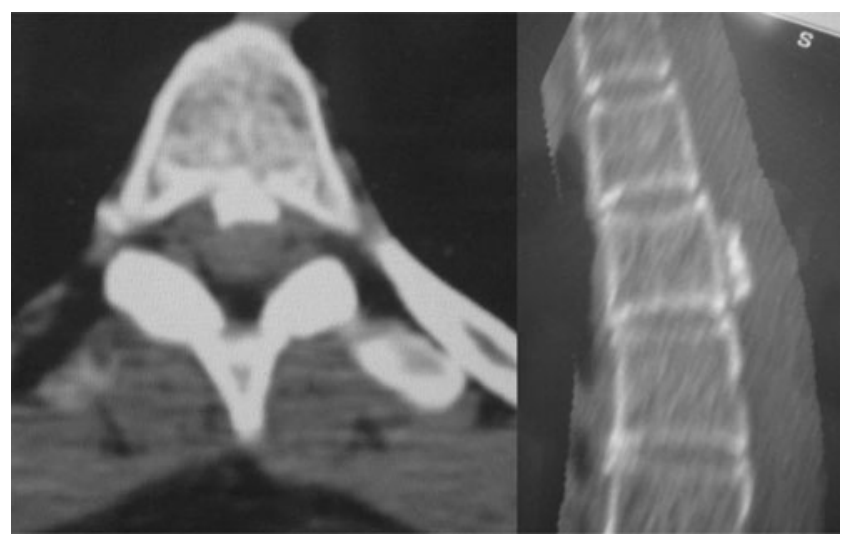

Fig. 3 Axial CT slice at T4 and sagittal reconstruction, demonstrating the intradural calcification posterior to and separated from the T4 vertebral body 


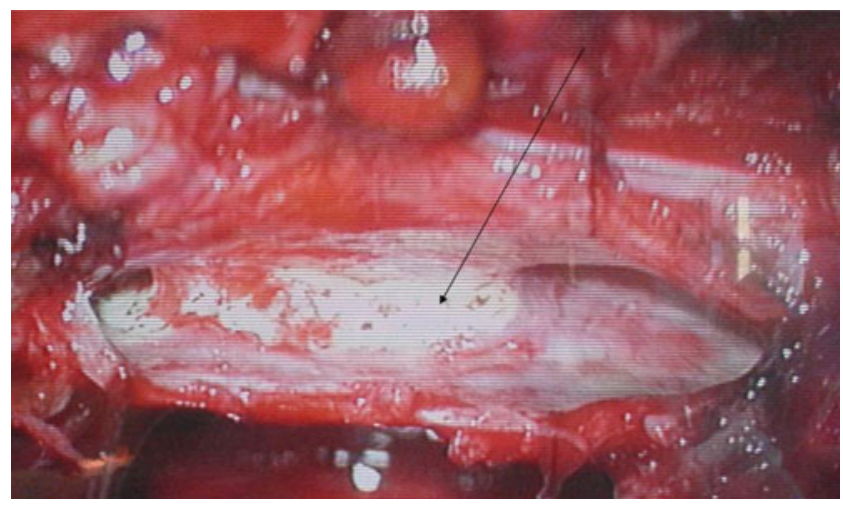

Fig. 4 After anterior paramedian dural opening: intraoperative view of the the calcification (arrow) between the arachnoid and dura, measuring about $15 \mathrm{~mm}$ in cranio-caudal diameter

removed in toto (Fig. 5), leaving a small arachnoid defect. Through this, a circumscribed bluish-reddish thin-walled capillary or venous anomaly, compatible with a venous cavernoma or hemangioma, bulged on the surface of a nerve root antero-lateral to the spinal cord (Fig. 6). It was coagulated with bipolars and thereby shrunk completely, so no histology tissue was taken.

After watertight dural closure, the T4 hemi-corpectomy was reconstructed by an expandable titanium cage and secured by a transpedicular stabilization T3 to T5 (Fig. 7).

No perioperative complication occurred, and the patient was mobilized on the second postoperative day. During 2 years after surgery, the patient showed minor improvement of hearing (hearing devices could finally be dropped), dysphagia, dysarthria, cerebellar ataxia, and tetraparesis. Muscle cramps and fasciculations improved significantly. Then, following a viral upper respiratory infection, deterioration with slowly progressive weakness of arms and legs was noted again. CSF analysis at that time was normal without signs of acute or chronic re-bleeding, and MRI

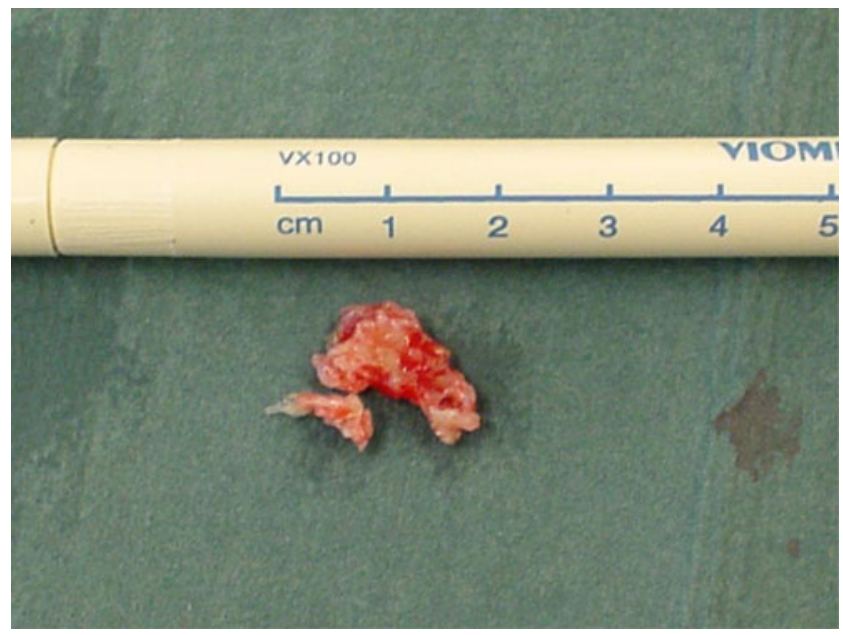

Fig. 5 Removed calcification

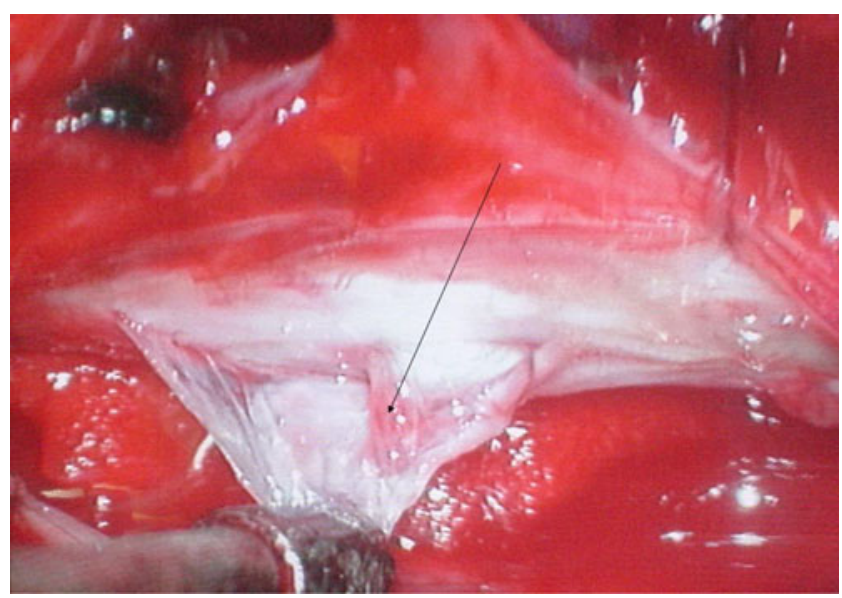

Fig. 6 Microsurgical view of the thin-walled vascular anomaly (suspected cavernoma or hemangioma; arrow) on an intradural nerve root at $\mathrm{T} 4$

revealed no further fluid collections and no arachnoid scarring with associated myelopathy (Fig. 8). Cranial MRI images at that time were unchanged compared to preoperative images (Fig. 9). Electroneuromyography showed slightly progressive upper and lower motoneuron dysfunction, and the electrophysiological revised El Escorial criteria were consistent with definite amyotrophic lateral sclerosis (ALS) [4]. However, clinical aspects (hypoacusia, ageusia, and cerebellar ataxia) and neuroimaging go beyond the exact diagnosis of ALS. Despite intensive physical therapy and even without further re-bleedings, the disease continued in a slow progression with severe cerebellar ataxia, muscle wasting, and weight loss to a body mass index of 16 . The patient finally died from pneumonia $31 / 2$ years after surgery.

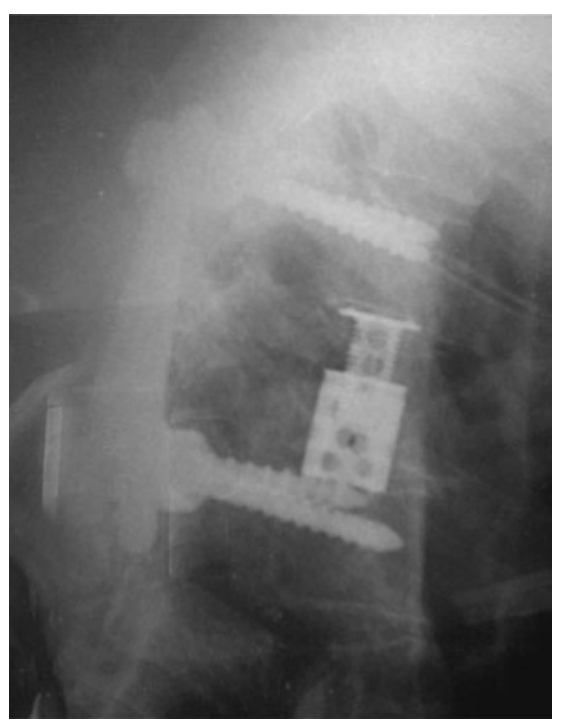

Fig. 7 Postoperative lateral thoracic spine radiograph, showing the reconstruction of the corpectomy by an expandable titanium cage and transpedicular fixation T3-5 

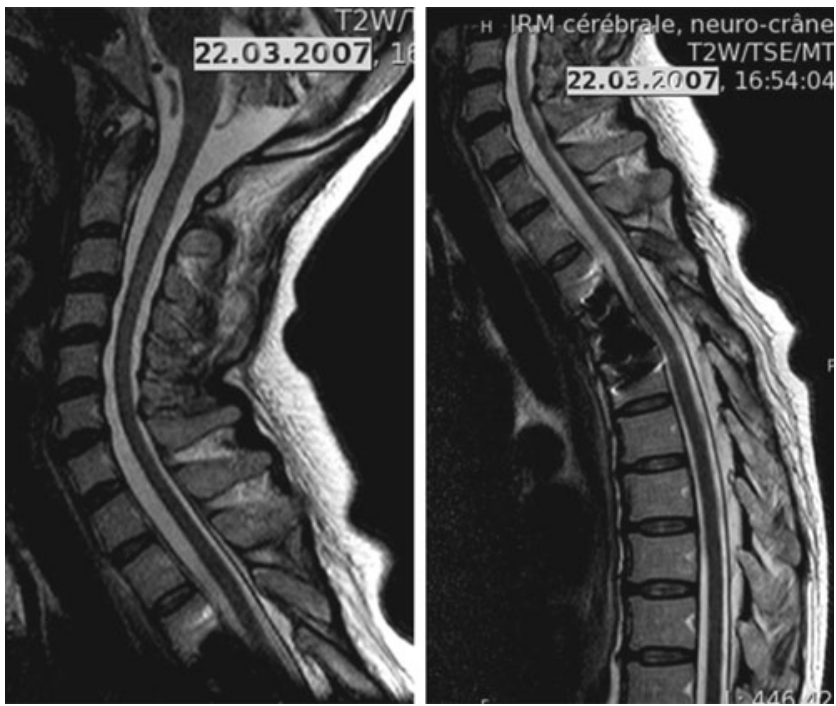

Fig. 8 Sagittal T2-weighted cervico-thoracic MRI 2 years postoperatively revealed no further fluid collections and no arachnoid scarring with associated myelopathy

\section{Discussion}

Superficial siderosis of the CNS has been consistently attributed to chronic subarachnoid hemorrhage, but the exact pathomechanism is poorly understood $[1,5,6,18]$. Excessive intrathecal iron from recurrent subdural bleeding leads to overloaded ferritin biosynthesis capacity of microglial cells; free radicals result and lipid peroxidation causes neuronal injury [10]. In contrast to glial cells, Schwann cells are spared, explaining the confinement of the disease to the CNS [9]. Due to its rarity, diagnosis of a superficial siderosis of the central nervous system requires a high index of suspicion $[5,18]$. The typical clinical presentation includes progressive sensorineural deafness, cerebellar ataxia, and pyramidal signs; dementia, bladder disturbance, anosmia, ageusia, anisocoria, and sensory signs have been observed as other typical symptoms in a systematic review $[6,14]$. The symptoms occur many years after the onset of bleeding $[1,18]$, and the diagnosis is usually made very late because of the rarity of the disease [6,17]. MRI is the neuroimaging of choice and shows a typical circumferential hypointensity rim around neural structures on T2-weighted and gradient echo sequences, indicative of hemosiderin deposition, particularly on the superior vermis and the cerebellar hemispheres $[1,3,20,21]$. MRI of the entire neuroaxis is always warranted in the search of a potential bleeding site [5, 6, 18]. CSF analysis may inconsistently reveal elevated red blood cells (RBC) or xanthochromia $[1,5]$. In our case, repeated CSF analysis never showed RBC or xanthochromia, but preoperative lumbar puncture revealed very high ferritin levels that lowered by half in the first year after operation.

In approximately $50 \%$ of the reported cases, no bleeding source was found, and untreated SS of the CNS is associated with a disabling progressive course $[1,5,6,20]$. Medical therapies with iron chelation or steroids have not been found effective $[6,9,24,27]$ except for a 6-month improvement reported in a single case [22].

In the other half of cases, the following bleeding sources have been identified $[1,6-8,11-13,15,16,26]$ : vascular tumors (mostly ependymomas), meningoceles or pseudomeningoceles after cervical nerve root avulsion, status post hemispherectomy, and vascular anomalies (cavernoma, arterio-venous malformation, arterio-venous fistula, aneurysm). The vascular anomaly on the intradural nerve root at T4 could have been the bleeding source in our case, as the micro-surgical appearance was compatible with an intradural extramedullary cavernous angioma or hemangioma (Fig. 6), as has been previously described [19, 23], too
Fig. 9 Axial T2-weighted cranial MRI images 2 years postoperatively show hypointense margins around the mesencephalon (arrow) and cerebellar hemispheres (arrow) without change compared to preoperative images

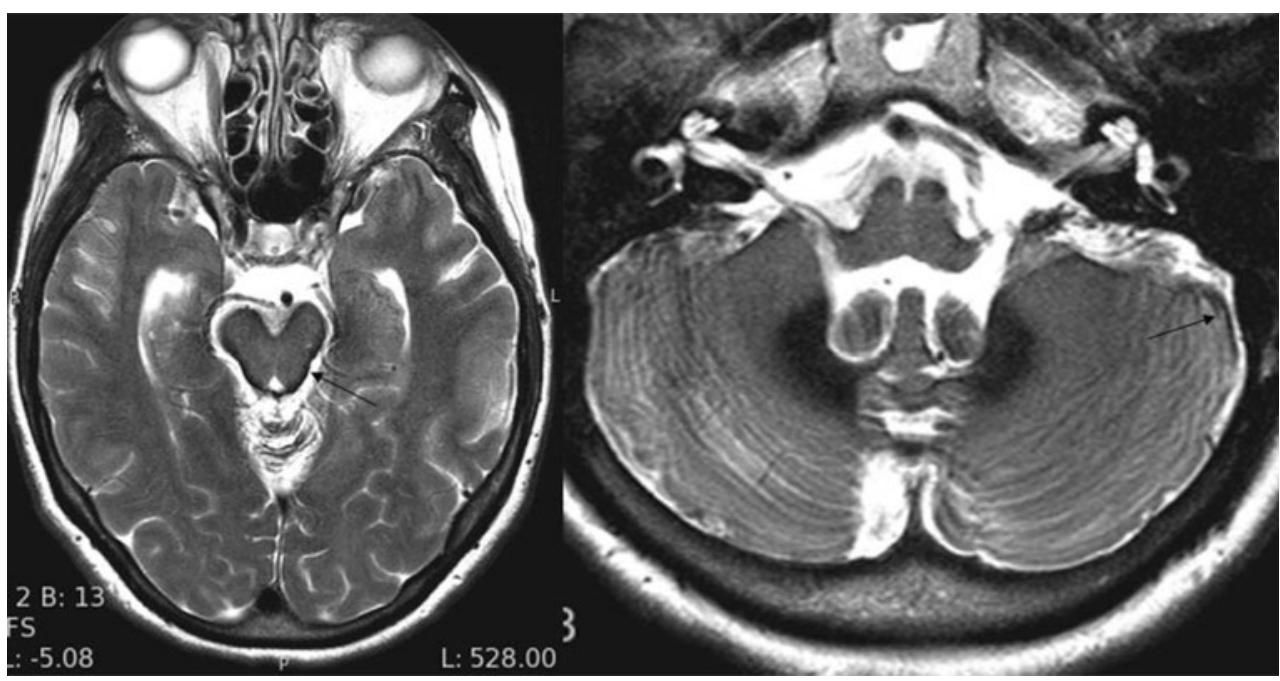


small to be detected on MRI or angiography at the time of presentation, or the calcification (Figs. 3 and 5) could have been a calcified meningioma functioning as bleeding source. As no histology was taken, the exact cause of bleeding remains open, but in any case, no further bleedings were observed after surgical elimination of the bleeding cause.

Surgical exclusion of the bleeding source is the only therapeutical option to stop the progression of the disease $[1,6,12,13,17,26]$.Yet, it remains unclear, whether the existing hemosiderin deposits in the leptomeninges and subpial layer will continue neuronal injury even after exclusion of the bleeding source [18]. Long-term outcome data in patients with surgical treatment of SS are scarce; most reports indicate no further progression of symptoms after surgery $[5,7,12,13,25,26]$, while others show that the disease may progress slowly even after surgery as the damage progression may be irreversible at the time of diagnosis [18]. Our case confirms that surgical exclusion of the bleeding site in SS of the CNS can stop further deterioration at least for a limited time. It also shows that even without further re-bleedings, secondary progression can occur in the form of a progressive upper and lower motoneuron disease, compatible with the clinical picture of ALS. This in return, suggests a common pathophysiological mechanism in the form of iron toxicity to the spinal cord including both the cortico-spinal tract and anterior horn cells, to cerebellar neurons and cranial nerves. To our knowledge, the association of SS and anterior horn-cell dysfunction has been expressed in one paper only [28], and this is the first report to describe a concomitant clinical picture of ALS. Further investigations and systematic electrophysiological studies in SS patients may help to better evaluate this association. Finally, it must remain open, if the patient coincidentally suffered from ALS as a second independent disease, but the statistical coincidence of ALS with SS of CNS is probably very low.

\section{Conclusion}

SS of the CNS is extremely rare, and a high index of suspicion is necessary for a timely recognition of the disease. The entire neuroaxis should be investigated with MRI for a bleeding source, the surgical exclusion of which stops symptom progression at least for a limited time. In our case of SS, surgical treatment of an intradural bleeding source at T4 led to mild clinical improvement for 2 years, after which, neurological deterioration mimicking ALS continued even without further re-bleeding.

Conflict of interest There is no conflict of interest.

\section{References}

1. Aquilina K, Kumar R, Lu J, Rawluk D (2005) Superficial siderosis of the central nervous system following cervical nerve root avulsion: the importance of early diagnosis and surgery. Acta Neurochir (Wien) 147(3):291-297

2. Bonito V, Agostinis C, Ferraresi S, Defanti CA (1994) Superficial siderosis of the central nervous system after brachial plexus injury. Case report. J Neurosurg 80(5):931-934

3. Bracchi M, Savoiardo M, Triulzi F, Daniele D, Grisoli M, Bradac GB, Agostinis C, Pelucchetti D, Scotti G (1993) Superficial siderosis of the CNS: MR diagnosis and clinical findings. AJNR 14(1):227-236

4. Brooks BR, Miller RG, Swash M, Munsat TL (2000) El Escorial revisited: revised criteria for the diagnosis of amyotrophic lateral sclerosis. Amyotroph Lateral Scler Other Motor Neuron Disord 1 (5):293-299

5. Cohen-Gadol A, Krauss W, Spinner R (2004) Delayed central nervous system superficial siderosis following brachial plexus avulsion injury. Report of three cases. Neurosurg Focus 16(5):E10

6. Fearnley J, Stevens J, Rudge P (1995) Superficial siderosis of the central nervous system. Brain 118(Pt 4):1051-1066

7. Haroun R, Li K, Rigamonti D (2000) Surgical resection of a cerebral arteriovenous malformation for treatment of superficial siderosis: case report. Surg Neurolo 53(6):554-558

8. Hug A, Hartmann M, Grau A (2004) Superficial siderosis of the CNS. 2 cases and a review of the literature. Nervenarzt 75(6):584 588

9. Koeppen A, Dentiger M (1988) Brain hemosiderin and superficial siderosis of the central nervous system. J Neuropathol Exp Neurol 47(3):249-270

10. Koeppen A, Borke R (1991) Experimental superficial siderosis of the central nervous system. I. Morphological observations. J Neuropathol Exp Neurol 50(5):579-594

11. Kole M, Steven D, Kirk A, Lownie S (2004) Superficial siderosis of the central nervous system from a bleeding pseudomeningocele. Case illustration. J Neurosurg 100(4):718

12. Konya D, Peker S, Ozgen S, Kurtkaya O, Necmettin Pamir M (2006) Superficial siderosis due to papillary glioneuronal tumor. J Clin Neurosci 13(9):950-952

13. Lévêque M, Mc Laughlin N, Bojanowski MW (2009) Secondary superficial siderosis of the central nervous system: report of three cases. Neurochirurgie 55(3):315-321

14. Levy M, Turtzo C, Llinas RH (2007) Superficial siderosis: a case report and review of the literature. Nat Clin Pract Neurol 3(1):54-58

15. Li KW, Haroun RI, Clatterbuck RE, Murphy K, Rigamonti D (2001) Superficial siderosis associated with multiple cavernous malformations: report of three cases. Neurosurgery 48(5):11471150, discussion 1150-1

16. Matsumoto S, Kang Y, Sato S, Kawakami Y, Oda Y, Araki M, Kawamura J, Uchida H (1998) Spinal meningeal melanocytoma presenting with superficial siderosis of the central nervous system. Case report and review of the literature. J Neurosurg 88(5):890 894

17. Messori A, De Bella P, Herber N, Loqullo F, Ruggiero M, Salvolini U (2004) The importance of suspecting superficial siderosis of the central nervous system in clinical practice. J Neurol Neurosurg Psychiatry 75(2):188-190

18. Miliaras G, Bostantjopoulou S, Argyropoulou M, Kyritsis A, Polyzoidis K (2006) Superficial siderosis of the CNS: report of three cases and review of the literature. Clin Neurol Neurosurg 108(5):499-502, Review

19. Nozaki K, Inomoto T, Takagi Y, Hashimoto N (2003) Spinal intradural extramedullary cavernous angioma. Case report. J Neurosurg 99(3 Suppl):316-319 
20. Offenbacher H, Fazekas F, Schmidt R, Kapeller P, Fazekas G (1996) Superficial siderosis of the central nervous system: MRI findings and clinical significance. Neuroradiology 38(Suppl 1):S51-S56

21. Pyhtinen J, Paakko E, Ilkko E (1995) Superficial siderosis in the central nervous system. Neuroradiology 37(2):127-128

22. River Y, Honigmann S, Gomori J, Reches A (1994) Superficial hemosiderosis of the central nervous system. Mov Disord 9(5):559-562

23. Roncaroli F, Scheithauer BW, Krauss WE (1999) Hemangioma of spinal nerve root. J Neurosurg 91(2 Suppl):175-180

24. Scheid R, Frisch S, Schroeter M (2009) Superficial siderosis of the central nervous system - treatment with steroids? J Clin Pharm Ther 34(5):603-605
25. Schievink W, Apostolides P, Spetzler R (1998) Surgical treatment of superficial siderosis associated with a spinal arteriovenous malformation. Case report. J Neurosurg 89(6):1029-1031

26. Shih P, Yang BP, Batjer HH, Liu JC (2009) Surgical management of superficial siderosis. Spine J 9(8):e16-e19

27. Stevens I, Petersen D, Grodd W, Poremba M, Dichgans J (1991) Superficial siderosis of the central nervous system. A 37-year follow-up of a case and review of the literature. Eur Arch Psychiatry Clin Neurosci 241(1):57-60

28. Turner B, Wills AJ (2002) Superficial siderosis associated with anterior horn cell dysfunction. J Neurol Neurosurg Psychiatry 72 (2):274-275 